\title{
IDENTIFICATION OF CERTAIN 4-MANIFOLDS WITH GROUP ACTIONS
}

\author{
RONALD FINTUSHEL AND PETER SIE PAO ${ }^{1}$
}

\begin{abstract}
If $M^{3}$ is an orientable 3-manifold with an $S^{1}$-action or is a Seifert fiber space, then the 4-manifold obtained by surgery along singular fibers in $M \times S^{1}$ can also be obtained by surgery in $V^{3} \times S^{1}$, where $V$ is a manifold related to $M$ but with fewer singular fibers. An application is given to Scharlemann's "exotic" $\left(S^{3} \times S^{1} \# S^{2} \times S^{2}\right)$ 's.
\end{abstract}

Group actions have been successfully used in studying a wide class of 3 and 4-manifolds [6] [9]. In this article we use circle actions to study the geometric properties of a family of 4-manifolds obtained from $M^{3} \times S^{1}$ by performing surgery. Specifically, let $M^{3}$ be a local $S^{1}$-manifold without $S E$-fibers [6], and in $M^{3} \times S^{1}$ perform surgery along $k$ circles of the form (exceptional orbit) $\times$ point. In Theorem 1 we show that the resulting manifold can be obtained by surgery along $k$ principal orbits in $V^{3} \times S^{1}$ where $V^{3}$ is a local $S^{1}$-manifold with structure simpler than that of $M$. (It has $k$ fewer exceptional orbits.)

The class of 3-manifolds with local $S^{1}$-action includes all the Seifert manifolds. If $M$ is a Seifert manifold with orbit space $S^{2}$ and $r$ exceptional fibers and if $k \geqslant r-2$, the above $V$ is a lens space. In particular, if $K$ is the dodecahedral space, surgery on an exceptional orbit in $K \times S^{1}$ yields an exotic 4-manifold studied by Scharlemann [10]. Theorem 1 implies that $W$ can be obtained by surgery along a principal orbit in some $S^{1} \times$ (lens space). We then use a theorem of Pao [8] to conclude that $W \# S^{2} \times S^{2}$ is diffeomorphic to $S^{3} \times S^{1} \# S^{2} \times S^{2} \# S^{2} \times S^{2}$.

1. Circle actions on 4-manifolds. In this section we review necessary preliminary material concerning the classification of circle actions on closed orientable 4-manifolds. Following [3] this is done in terms of a weighted 3-manifold which consists of the orbit space together with information about the orbit types.

If $S^{1}$ acts locally smoothly on the closed oriented 4-manifold $W$ the orbit space $W^{*}$ is an oriented 3-manifold. The orbits in $W$ which have nontrivial finite cyclic isotropy groups are called exceptional, and their image $E^{*}$ in $W^{*}$

Presented to the Society April 1, 1977; received by the editors February 17, 1977 and, in revised form, May 2, 1977.

AMS (MOS) subject classifications (1970). Primary 57E25, 55D99.

'This research was partially supported by N.S.F. Grants. 
consists of a finite collection of circles and open arcs. Each component of $E^{*}$ has constant orbit type and is oriented and assigned Seifert invariants $(\alpha, \beta)$. Thus if we identify a neighborhood of a point $x^{*} \in E^{*}$ with $I \times D^{2}, I \subseteq E^{*}$, then the $S^{1}$-submanifold of $W$ over $I \times D^{2}$ is equivalent to a product action $I \times\left(D^{2} \times S^{1}\right)$ where $S^{1}$ acts on $D^{2} \times S^{1}$ with Seifert invariants $(\alpha, \beta)[6]$.

If $F^{*}$ denotes the image in $W^{*}$ of the fixed point set then $E^{*} \cup F^{*}$ is the disjoint union of $\partial W^{*}$ and a collection of closed arcs, circles, and isolated points. To each closed arc in $E^{*} \cup F^{*}$ an ordered pair of the integers 0 and -1 is also assigned, but this can be ignored for the purposes of this paper.

There is one further invariant which is important for classifying the $S^{1}$-action, namely, the characteristic class. It is defined as follows. Let $Q^{*}$ be a regular neighborhood of the circles in $E^{*} \cup F^{*}$ and let $N^{*}$ be a regular neighborhood of the remainder $S^{*}$ of $E^{*} \cup F^{*}$. The restriction of the orbit map over $\mathrm{Cl}\left(W^{*}-\left(Q^{*} \cup N^{*}\right)\right)$ is a principal $S^{1}$-bundle projection which is trivial over $\partial Q^{*}$. This bundle can be extended over $Q^{*}$ by gluing in $Q^{*} \times S^{1}$ with a bundle isomorphism. There are infinitely many choices for the gluing bundle isomorphism, but a specific gluing map can be chosen in a natural way. (We refer the reader to $[3, \S 9]$ for details.) Thus we determine a principal $S^{1}$-bundle over $\mathrm{Cl}\left(W^{*}-N^{*}\right)$; let $\chi^{\prime} \in H^{2}\left(\mathrm{Cl}\left(W^{*}-N^{*}\right)\right)$ be its Euler class. By Poincaré duality

$$
\begin{aligned}
H^{2}\left(\mathrm{Cl}\left(W^{*}-N^{*}\right)\right) & \approx H_{1}\left(\mathrm{Cl}\left(W^{*}-N^{*}\right), \partial \mathrm{Cl}\left(W^{*}-N^{*}\right)\right) \\
& \approx H_{1}\left(W^{*}, N^{*}\right) \approx H_{1}\left(W^{*}, S^{*}\right) .
\end{aligned}
$$

The Poincaré dual of $\chi^{\prime}$, say $\chi \in H_{1}\left(W^{*}, S^{*}\right)$, is called the characteristic class of the $S^{1}$-action on $W$.

The weighted orbit space of the $S^{1}$-action on $W$ consists of the oriented orbit space $W^{*}$, the characteristic class $\chi \in H_{1}\left(W^{*}, S^{*}\right)$, and $E^{*} \cup F^{*}$ along with the orbit data described above. An isomorphism $f: W_{1}^{*} \rightarrow W_{2}^{*}$ of weighted orbit spaces is an orientation-preserving homeomorphism which preserves orbit data and satisfies $f\left(\chi_{1}\right)=\chi_{2}$ where $\chi_{i} \in H_{1}\left(W_{i}^{*}, S_{i}^{*}\right)$ is the characteristic class.

Equivariant Classification Theorem [3]. Oriented closed 4-manifolds with locally smooth $S^{1}$-actions are orientation-preserving equivariantly homeomorphic if and only if they have isomorphic weighted orbit spaces.

One sees from the constructions of [2] and [3] that each such action is equivalent to a smooth action. Also it follows easily from the proof of the classification theorem that if $W$ and $W^{\prime}$ are smooth $S^{1}$-manifolds with isomorphic weighted orbit spaces there is a (not necessarily equivariant) PL homeomorphism $W \rightarrow W^{\prime}$. Thus $W$ and $W^{\prime}$ are diffeomorphic since PL $=$ DIFF in these low dimensions.

2. Surgery on $M^{3} \times S^{1}$. One of the commonly used techniques of constructing interesting 4-manifolds consists of performing surgeries on a known 4-manifold along some properly chosen circles. The known 4-manifold 
is frequently taken to be a bundle over $S^{1}$-with fiber a 3-manifold [1], [5], [9], [10]. In this section we study a family of 4-manifolds constructed in this way.

A local $S^{1}$-action on a space $X$ is a decomposition of $X$ into points and circles such that each decomposition element has a neighborhood admitting an effective $S^{1}$-action with the elements of the decomposition as orbits. A local $S^{1}$-action on a 3 -manifold is characterized by its orbit invariants. We shall be concerned here with orbit invariants of the form $\{b ;(\varepsilon, g, \bar{h}, 0)$; $\left.\left(\alpha_{1}, \beta_{1}\right), \ldots,\left(\alpha_{r}, \beta_{r}\right)\right\}$, which can be explained briefly as follows (see [6]). If $M$ is a local $S^{1}$-3-manifold with the above invariants, its orbit (decomposition) space is a 2 -manifold $M^{*}$ of genus $g$ with $\bar{h}$ boundary components, the images of $\bar{h}$ circles of fixed points in $M . M^{*}$ is orientable if $\varepsilon=o_{1}$ or $o_{2}$, nonorientable if $\varepsilon=n_{1}, n_{2}, n_{3}$ or $n_{4}$. There are $r$ exceptional orbits in $M$ with Seifert invariants $\left(\alpha_{1}, \beta_{1}\right), \ldots,\left(\alpha_{r}, \beta_{r}\right)$. The integer $b$ and $\varepsilon$ classify up to weak equivalence an $S^{1}$-bundle over Int $M^{*}$ which is obtained by canonically replacing tubular neighborhoods of exceptional orbits with tubular neighborhoods of principal orbits. The local action is an action if and only if $\varepsilon=o_{1}$ or $n_{2}$, and in this case the associated $S^{1}$-bundle is principal and $b$ is its Euler class.

Now let $M$ be a 3 -manifold with the local $S^{1}$-action $\{b ;(\varepsilon, g, \bar{h}, 0)$; $\left.\left(\alpha_{1}, \beta_{1}\right), \ldots,\left(\alpha_{r}, \beta_{r}\right)\right\}$, and let the 2-manifold $M^{*}$ be its orbit space. The 4-manifold $N=M \times S^{1}$ inherits a product local $S^{1}$-action, trivial in the $S^{1}$-factor. The orbit space of this local action is $N^{*}=M^{*} \times S^{1}$, and $E^{*}$ consists of $r$ oriented circles with Seifert invariants $\left(\alpha_{1}, \beta_{1}\right), \ldots,\left(\alpha_{r}, \beta_{r}\right)$. Let $C \subseteq N$ be any circle orbit, and let $T$ be a tube of $C$; so $T \cong S^{1} \times D^{3}$. Performing a surgery on $N$ along $C$ removes $T$ and fills in a copy of $D^{2} \times S^{2}$. It is easily seen that this cutting and pasting can always be done equivariantly; i.e. we can define an $S^{1}$-action on $D^{2} \times S^{2}$ so that the attaching map is $S^{1}$-equivariant (cf. [9, §3]). Therefore, after performing surgeries on $N$ along circle orbits, the resulting manifold again has a local $S^{1}$-action. On the orbit space level the surgery replaces the orbit space of $T$, a 3-cell, with the orbit space of $D^{2} \times S^{2}$, a 3-cell or $S^{2} \times I$. If $C$ is an exceptional orbit of type $\left(\alpha_{i}, \beta_{i}\right)$ the orbit space of $D^{2} \times S^{2}$ will be a 3-cell. The effect of this surgery on the orbit space is to replace the circle in $E^{*}$ corresponding to $\left(\alpha_{i}, \beta_{i}\right)$ by the circle

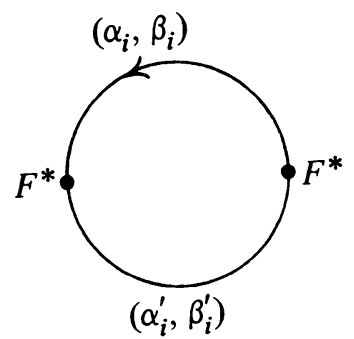

or the arc 


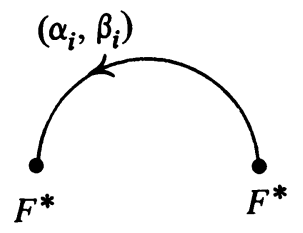

In the second case the surgery has replaced orbits corresponding to the missing arc by principal orbits having trivial isotropy.

THEOREM 1. Let $M^{3}$ be the local $S^{1}$-manifold $\{b ;(\varepsilon, g, \bar{h}, 0)$; $\left.\left(\alpha_{1}, \beta_{1}\right), \ldots,\left(\alpha_{r}, \beta_{r}\right)\right\}$ and let $W$ be a 4-manifold obtained from $M \times S^{1}$ by performing surgeries on the circles $C_{i} \times t_{i}, i=k+1, \ldots, r$, where $C_{i}$ is the exceptional orbit in $M$ of type $\left(\alpha_{i}, \beta_{i}\right)$ and $t_{i} \in S^{1}$. Then there is a local $S^{1}$-manifold $V^{3}=\left\{a ;(\varepsilon, g, \bar{h}, 0) ;\left(\alpha_{1}, \beta_{1}\right), \ldots,\left(\alpha_{k}, \beta_{k}\right)\right\}$ such that $W$ is diffeomorphic to a 4-manifold obtained from $V \times S^{1}$ by doing surgeries along $r-k$ principal orbits.

Proof. Consider first the case where $M$ is an oriented $S^{1}$-manifold ( $\varepsilon=$ $\left.o_{1}\right)$. It follows from the above discussion that $W$ is an $S^{1}$-manifold with orbit space $W^{*}=M^{*} \times S^{1}, E^{*} \cup F^{*}$ consists of $\partial W^{*}, k$ oriented circles in $E^{*}$ with Seifert invariants $\left(\alpha_{1}, \beta_{1}\right), \ldots,\left(\alpha_{k}, \beta_{k}\right)$, and $r-k$ circles and arcs in $E^{*} \cup F^{*}$ as described above. Viewing $M^{*} \times S^{1}$ as obtained from $M^{*} \times I$ by identifying $M^{*} \times 0$ with $M^{*} \times 1$, we picture $W^{*}$ in Figure 1 , leaving unlabelled the $r-k$ circles and arcs of $E^{*} \cup F^{*}$.

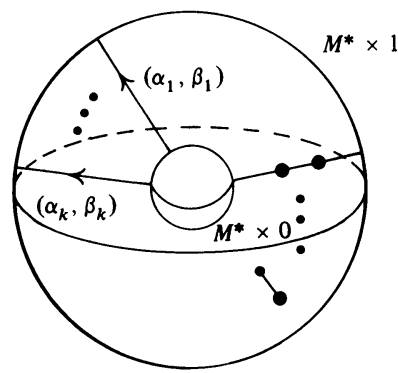

FiguRE 1

Applying the replacement trick of $[9, \S 2]$ to these $r-k$ components of $E^{*} \cup F^{*}$ alters the $S^{1}$-action on $W$ so that the new orbit space $W_{1}^{*}$ consists of $W^{*}$ without the above $r-k$ circles and arcs and with $0 \leqslant j \leqslant r-k$ open 3 disks removed and $r-k-j$ pairs of fixed points in int $W_{1}^{*}$ (Figure 2).

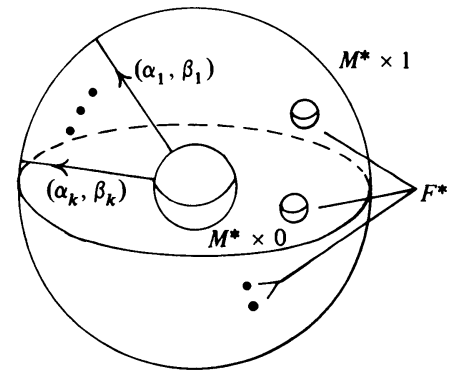

FigURE 2 
The manifold $W$ is determined up to diffeomorphism by this weighted orbit space and the characteristic class of the action. The dual characteristic class $\chi^{\prime}$ lies in $H^{2}\left(W^{*}-S^{*}\right)$, which is embedded in the short exact sequence

$$
0 \rightarrow H^{2}\left(M^{*} \times S^{1}\right) \rightarrow H^{2}\left(W^{*}-S^{*}\right) \rightarrow \operatorname{ker} \delta \rightarrow 0
$$

where $\delta$ is the Mayer-Vietoris coboundary arising from the sequence for $M^{*} \times S^{1} \cong\left(W^{*}-S^{*}\right) \cup\left(\dot{\cup}_{t} D^{3}\right), t=j+2(r-k-j)$. Since ker $\delta \subseteq$ $H^{2}\left(\cup_{t} S^{2}\right)$, the sequence splits and we identify $H^{2}\left(W^{*}-S^{*}\right)=H^{2}\left(M^{*} \times\right.$ $\left.S^{1}\right) \oplus \operatorname{ker} \delta$ and $\chi^{\prime}=(\xi, \eta)$.

We may choose circles $\left\{S_{i}\right\}$ in $M^{*}$ which generate $H_{1}\left(M^{*}\right)$ and which do not intersect $E^{*} \cup F^{*}$. The various applications of surgery and the replacement trick performed on $M \times S^{1}$ to obtain $W$ do not alter the trivial principal orbit bundle over each $S_{i} \times S^{1}$. Thus if $V^{*}=M^{*} \times 1$ in Figure $2, \xi$ is determined by $\xi \mid V^{*}$ which in turn is determined by the integer $a$, where the $S^{1}$-submanifold $V$ of $W$ over $V^{*}$ has orbit invariants $\left\{a ;\left(o_{1}, g, \bar{h}, o\right)\right.$; $\left.\left(\alpha_{1}, \beta_{1}\right), \ldots,\left(\alpha_{k}, \beta_{k}\right)\right\}$. The other component $\eta$ of $\chi^{\prime}$ is determined by the principal orbit bundles over the components of $\dot{U}_{t} S^{2}$, all of which arise due to the replacement trick. Over each of the $j 2$-spheres which are boundaries of collar neighborhoods of 2-spheres in $F^{*}$, the principal orbit bundle is trivial. The remaining $2(r-k-j)$ 2-spheres are the boundaries of regular neighborhoods of single point components of $F^{*}$. They are introduced in pairs by the replacement trick and the Euler numbers of the bundles over the associated 2-spheres occur in a $\pm_{1}$-pair. It then follows that the isomorphism type of the weighted orbit space $W_{1}^{*}$, and therefore the diffeomorphism type of $W$, depends only on the orbit data of $W_{1}^{*}$ and the $S^{1}$-action over $V^{*}$. For this reason we call $V^{*}$ a characteristic surface of $W_{1}^{*}$.

Let $C_{1}^{\prime}, \ldots, C_{k-r}^{\prime}$ be principal orbits in $V \times S^{1}$. By performing equivariant surgeries in $V \times S^{1}$ with properly chosen framings we can construct an $S^{1}$-manifold $W_{2}$ whose weighted orbit space $W_{2}^{*}$ is exactly $W_{1}^{*}$. In $V^{*} \times S^{1}$ there is a $V^{*} \times t$ which is not affected by the surgeries, and this gives rise to a characteristic surface $V_{2}^{*} \subseteq W_{2}^{*}$. The action over $V_{2}^{*}$ is just that on $V$, hence $W$ and $W_{2}$ are diffeomorphic.

If $\varepsilon=n_{2}$ a similar argument applies. Although the equivariant classification theorem of [3] does not generally cover $S^{1}$-actions on nonorientable 4-manifolds, the extension to the particular case at hand is trivial.

In case $\varepsilon \neq o_{1}, n_{2}$ the replacement trick stil[ gives another local $S^{1}$-action to $W$ with orbit space $W_{1}^{*}$ as in Figure 2. The equivariant classification theorem has an analogue in this particular situation. Using notation of $\S 1$, one obtains over $\mathrm{Cl}\left(W_{1}^{*}-N^{*}\right)$ a (nonorientable) $S^{1}$-bundle which is classified by its first Stiefel-Whitney class (viewed as a homomorphism $\omega: \pi_{1}\left(W_{1}^{*}\right)=\pi_{1}\left(\mathrm{Cl}\left(W_{1}^{*}-\right.\right.$ $\left.\left.\left.N^{*}\right)\right) \rightarrow \mathbf{Z}_{2}\right)$ and by its primary obstruction $o_{2} \in H^{2}\left(\mathrm{Cl}\left(W_{1}^{*}-N^{*}\right) ; \mathbf{Z}^{t}\right)$ where coefficients are twisted by $\omega$. The pair of invariants $\left(\omega, o_{2}\right)$ replaces the characteristic class, and the classification then proceeds as in $\S 1$ except that unoriented Seifert invariants $(1 \leqslant \beta \leqslant \alpha / 2)$ are required (cf. [11]). If a 
characteristic surface is chosen as before, then $\left(\omega, o_{2}\right)$ can be obtained from the corresponding invariants over $V^{*}$; so the earlier proof applies to the general situation.

3. Scharlemann's manifolds. Let $K$ be the dodecahedral space, and in $K \times S^{1}$ let $C \times$ point be an embedded circle representing a noncentral element of $\pi_{1}(K)$. There are two framings for surgery along $C \times$ point and we denote the resulting 4-manifolds by $W_{c}$ and $W_{c}^{\prime}$, distinguished by $w_{2}\left(W_{c}\right)$ $=0$ and $w_{2}\left(W_{c}^{\prime}\right) \neq 0$. It is known that for all such $C, W_{c}^{\prime}$ is diffeomorphic to $S^{3} \times S^{1} \# C P^{2} \#-C P^{2}$. There are 118 noncentral elements in $\pi_{1}(K)$, and the corresponding manifolds $W_{c}$ were introduced by M. Scharlemann [10] to construct fake homotopy structures on $S^{3} \times S^{1} \# S^{2} \times S^{2}$. We address the question of whether the manifolds $W_{c}$ are diffeomorphic to $S^{3} \times S^{1} \# S^{2} \times$ $S^{2}$ [4, Problem 4.15].

It is well known that $K$ carries the $S^{1}$-action with orbit data $\{-1$; $\left.\left(o_{1}, 0,0,0\right) ;(2,1),(3,1),((5,1))\right\}$. If $C$ is an exceptional orbit in $K$, it represents a noncentral element in $\pi_{1}(K)$, and it follows from Theorem 1 that $W_{c}$ can be obtained from $L \times S^{1}$, for some lens space $L=\left\{a ;\left(o_{1}, 0,0,0\right)\right.$; $\left.\left(\alpha_{1}, \beta_{1}\right),\left(\alpha_{2}, \beta_{2}\right)\right\}$, by performing surgery along a principal orbit. In [8, III. 3 and III. 5] it is shown that surgery on $L \times S^{1}$ along an exceptional orbit $C^{\prime}$ yields $S^{3} \times S^{1} \# S^{2} \times S^{2}$. After performing another surgery, on $C$, which can now be viewed as lying in $S^{3} \times S^{1} \# S^{2} \times S^{2}$, we obtain $S^{3} \times$ $S^{1} \# S^{2} \times S^{2} \# S^{2} \times S^{2}$. On the other hand, after doing surgery on $C$, the orbit $C^{\prime}$ becomes homotopically trivial in $W_{c}$; so by general position $C^{\prime}$ bounds an immersed disk with isolated double points which can be piped to the boundary along arcs and eliminated. Thus $C^{\prime}$ bounds a smooth 2-disk in $W_{c}$, and surgery on $C^{\prime}$ yields $W_{c} \# S^{2} \times S^{2}$. Since $C$ and $C^{\prime}$ are disjoint the order of the surgeries is irrelevant and we obtain

$$
W_{c} \# S^{2} \times S^{2}=S^{3} \times S^{1} \# S^{2} \times S^{2} \# S^{2} \times S^{2} .
$$

For arbitrary choices of circles $C_{1}, C_{2}$ in $K \times S^{1}$ representing noncentral elements of $\pi_{1}(K)$, the above argument shows

$$
W_{c_{1}} \# S^{2} \times S^{2}=W_{c_{2}} \# S^{2} \times S^{2} .
$$

In conclusion:

THEOREM 2. If $C$ is a simple closed curve in $K$ representing a noncentral element of $\pi_{1}(K)$ then $W_{c} \# S^{2} \times S^{2}$ is diffeomorphic to $S^{3} \times S^{1} \# 2\left(S^{2} \times\right.$ $\left.S^{2}\right)$.

Of course, we would like to show that $W_{c}=S^{3} \times S^{1} \# S^{2} \times S^{2}$, but explicit computations following the proof of Theorem 1 put this result slightly out of reach. For example, if $C$ is the exceptional orbit in $K$ with Seifert invariants $(5,1)$ then $W_{c}$ carries a circle action with orbit space equal to $S^{2} \times S^{1}$ minus an open 3-disk as in Figure 3. 


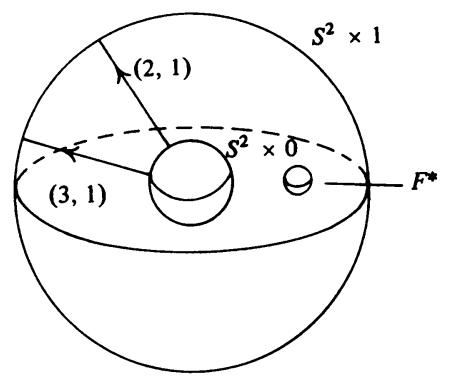

Figure 3

The characteristic class of this action is $\chi=0$. The manifold $S^{3} \times S^{1} \# S^{2}$ $\times S^{2}$ carries a circle action with exactly the same orbit space but with $\chi=-1$.

\section{REFERENCES}

1. S. E. Cappell and J. L. Shaneson, Some new four-manifolds, Ann. of Math. (2) 104 (1976), 61-72.

2. R. Fintushel, Circle actions on simply connected 4-manifolds, Trans. Amer. Math. Soc. 230 (1977), 147-171.

3. Classification of circle atoms on 4 manifolds, Trans. Amer. Math. Soc. (to appear).

4. R. Kirby, Problems in low dimensional topology, Proc. AMS Summer Institute in Topology, Stanford, 1976.

5. B. Mazur, Symmetric homology spheres, Illinois J. Math. 6 (1972), 245-250.

6. P. Orlik and F. Raymond, On 3-manifolds with local SO(2)-action, Quart. J. Math. Oxford Ser. 20 (1969), 143-160.

7. Actions of the torus on 4-manifolds. II, Topology 13 (1974), 89-112.

8. P. S. Pao, The topological structure of 4 manifolds with effective torus actions. I, Trans. Amer. Math. Soc. 227 (1977), 279-317; II, Illinois J. Math. (to appear).

9. Nonlinear circle actions on the 4-sphere and a notion on knot theory, Topology (to appear).

10. M. Scharlemann, Constructing strange manifolds with the dodecahedron space, Duke Math. J. 43 (1976), 33-40.

11. H. Seifert, Topologie dreidimensionaler gefaserter Räume, Acta Math. 60 (1933), 147-238.

Department of Mathematics, Tulane University, New Orleans, Lousiana 70118 (Current address of Ronald Fintushel)

Department of Mathematics, University of Georgia, Athens, Georgia 30602

Current address (P. S. Pao): School of Mathematics, Institute for Advanced Study, Princeton, New Jersey 08540 